\title{
Herpes Simplex Virus (HSV) in the Differential for Fulminant Hepatic Failure
}

\author{
Kevin Ergle, Lawrence Caruso, Martha Burt, Bobby Desai, Rohit Patel \\ University of Florida Health, Gainesville, USA \\ Email: bdesai@ufl.edu
}

Received 26 January 2015; accepted 8 February 2015; published 13 February 2015

Copyright (C) 2015 by authors and Scientific Research Publishing Inc.

This work is licensed under the Creative Commons Attribution International License (CC BY). http://creativecommons.org/licenses/by/4.0/

(c) (7) Open Access

\begin{abstract}
Following an elective transphenoidal resection of a pituitary tumor, a 66-year-old Hispanic male acutely developed fulminant hepatic failure and severe coagulopathy. He received parenteral corticosteroids the day prior to surgery, and was first noted to have significant coagulopathy intraoperatively. Despite aggressive workup and treatment for fulminant hepatic failure, the patient developed multiorgan failure by post-operative day 2 , and expired on post-operative day 3 . On post-mortem examination, hemorrhagic necrosis of the liver was noted and microscopic examination revealed Cowdry type A inclusions, consistent with Herpes Simplex Virus (HSV) infection. This diagnosis was subsequently confirmed by immunehistochemistry. Fulminant hepatic failure due to HSV is a rare but highly fatal disease if untreated. Most case reports include only immunocompromised or pregnant patients. However, the lack of clinical suspicion in this previously healthy patient may have delayed prompt treatment with antiviral agents. It is important to raise the awareness of this rare and life-threatening, but potentially treatable, etiology when clinicians are faced with acute idiopathic fulminant hepatic failure.
\end{abstract}

\section{Keywords}

Fulminant Hepatic Failure, HSV

\section{Introduction}

Fulminant hepatic failure affects approximately 2000 people in the United States annually. Drug-related causes account for more than $50 \%$ of the cases of hepatic failure, with viruses and autoimmune causes representing much of the remainder of the cases. HSV is fairly ubiquitous in the United States, with seroprevalence among those 14 - 49 years of age at $62 \%$ for HSV-1 and $17.2 \%$ for HSV-2 [1]. However, HSV-induced hepatic failure is rare, and has been estimated to account for approximately $1 \%$ of all causes of fulminant hepatic failure [2]. De- 
spite being first described in 1969 [3], herpes simplex hepatitis has been an often unrecognized form of fulminant hepatic failure which is rapidly (within days) fatal and with mortality rate approaching 90\% [4]. Most case reports have shown to be in patients who are immunocompromised, frequently pregnant women in 3rd trimester, newborns, or patients otherwise immunosuppressed either by HIV or by immunomodulatory agents treating other diseases. Here we present a case of HSV-induced fulminant hepatic failure in an otherwise immunocompetent male.

\section{Case Presentation}

We present a case of a 66-year-old immunocompetent Hispanic male who developed herpes hepatitis after surgery for removal of a pituitary mass. The patient had a past medical history of hypertension, dyslipidemia, and skin cancer. He had no known history of immunosuppression or opportunistic infections. He presented to neurosurgery clinic with complaint of 10 days of headache and diplopia, where physical exam revealed right-sided cranial nerve (CN) III palsy and diplopia with left lateral gaze. He had been previously diagnosed with a pituitary mass four years prior, which had been treated with bromocriptine up to that point. CT scan and MRI showed $2.8 \mathrm{~cm} \times 1.8 \mathrm{~cm}$ sella mass extending into the sphenoid sinus with lateral extension into the cavernous sinus. Surgical intervention with transnasal transphenoidal hypophysectomy with stereotactic guidance and abdominal fat graft was planned.

One day preoperatively, the patient was admitted to the hospital and started on hydrocortisone $100 \mathrm{mg}$ IV q8h. Laboratory evaluation at that time showed INR 1.1, PTT 27, Platelets 190, Hematocrit 41, with pituitary panel revealing normal random serum cortisol but low prolactin, TSH, T3, T4, and testosterone levels. The operation proceeded uneventfully. However, toward the end of the procedure the surgeon noted persistent oozing in mucosa and bone edges that was difficult to control. Coagulation studies were sent intra-operatively and were markedly abnormal. Patient was given fresh frozen plasma (FFP) and vitamin K for elevated INR and PTT. Eventually hemostasis was achieved and patient was extubated in the OR. He was following complex commands and moving all 4 extremities before he was taken to the PACU in the afternoon. Physical exam did not show any jaundice or abdominal pain and patient had normal vital signs without a fever. Due to his intra-operative course additional coagulation studies were ordered that showed significant thrombocytopenia in addition to elevated prothrombin times. He was subsequently transfused platelets, additional FFP and packed red blood cells, a hematology consult was ordered and patient was planned for an intensive care unit (ICU) stay to fully work up his coagulopathy.

The patient developed hypoxic respiratory failure several hours after transfer to the Neurosurgical ICU and was emergently intubated, after which his hypoxia improved. On post-op day 1, patient was noted to have transaminitis which was steadily rising and coagulopathy causing increased bleeding and anemia despite multiple units of FFP, platelets, and packed red blood cells. Additionally, the patient developed acute renal failure and metabolic acidosis. He was evaluated by the hepatology service and was not felt to be a candidate for transplant at that time, due to instability and possibly active infection. Despite aggressive intervention, including broad-spectrum antibiotics for concern for sepsis, the patient's condition continued to decline and he suffered PEA arrest on post-operative day 2. He had return of spontaneous circulation after two rounds of CPR and epinephrine, and was started on vasopressors to maintain blood pressure and Continuous Veno-Venous Hemofiltration (CVVH) was initiated for worsening renal function. Laboratory evaluation was unable to identify the source of his fulminant hepatic failure and coagulopathy. On post-operative day 3, after continued decline of the patient's function, the family made the decision to withdraw care, and the patient expired. Specific laboratory values for the patient are described in Table 1.

Post-mortem examination revealed coagulopathy with striking thrombocytopenia without evidence of disseminated intravascular coagulation (DIC) likely due to massive hemorrhagic hepatic viral necrosis and a focal area of lung with HSV viral infection. Other findings included hemorrhagic bowel mucosa with bilateral serosanguinous pleural effusions, focal acute basal ganglia hemorrhage, and focal subarachnoid hemorrhage along with early ischemic changes. The right lower lung lobe had a $4 \mathrm{~cm}$ ill-defined firm abscess microscopically composed of acute inflammation, hemorrhage, necrosis, and distinctive viral cytopathic effect similar to that in the liver. Although the viral changes were observed throughout the liver parenchyma, the viral changes in the lungs were limited to the right lower lung lobe abscess. The remainder of the lung parenchyma had mild to moderate congestion, but no other significant inflammation or necrosis. None of the other organs or tissues 
Table 1. Lab values.

\begin{tabular}{|c|c|c|c|c|c|c|c|}
\hline Post-op day & INR & PTT & Platelets & AST & ALT & Bilirubin (total) & WBC \\
\hline Pre-op & 1.1 & 27 & 190 & Unknown & Unknown & Unknown & $16.6^{*}$ \\
\hline 0 & 1.9 & 50 & 12 & 3736 & 3081 & 2 & 9.2 \\
\hline 1 & 1.9 & 62 & 20 & 8647 & 8399 & 3.7 & 6.1 \\
\hline 2 & 3.6 & 80 & 39 & 12,505 & 13,655 & 4.9 & 5.3 \\
\hline 3 & $>8.0$ & $>150$ & 20 & 4651 & 6823 & 2.4 & 1 \\
\hline
\end{tabular}

Note that patient received multiple units of FFP, platelets, and pRBCs during hospital stay. *Pre-op patient was on steroids.

examined showed any signs of viral infection, inflammation or necrosis, including the central nervous system, gastrointestinal tract, larynx and trachea. Viral inclusions were presents and presence of HSV was confirmed with immunohistochemical stain. The liver was slightly enlarged (2128 grams, reference range, 1500 - 1800 grams), and had firm, dark red parenchyma. Microscopically, the liver parenchyma was diffusely necrotic (Figure 1), with minimal cellular detail, and with abundant parenchymal hemorrhage. Many of the viable hepatocytes had enlarged, deeply basophilic nuclei with the characteristic "ground glass" chromatin pattern that in some instances resemble ground glass nuclear inclusions (Figure 2). Other nuclei had the characteristic punctate, granular chromatin pattern (Figure 3). Although the histologic features are quite characteristic of herpes virus infection, this was confirmed with an HSV immunohistochemical stain (Figure 4). These nuclear changes are commonly refered to as "viral cytopathic effect," and were observed in essentially all sections of the liver examined.

\section{Discussion}

The low prevalence of HSV-induced hepatic failure leads to low clinical suspicion and the diagnosis often being missed. One review showed that only $26 \%$ of non-pregnant and $33 \%$ of pregnant patients with HSV hepatitis were diagnosed antemortem [5], with others estimating the antemortem diagnosis only as high as $42 \%$ [6]. However, HSV-induced FHF remains one of the few treatable causes of fulminant hepatic failure. It has been shown that the percentage of patients progressing to death or liver transplantation from HSV hepatitis can be reduced from $88 \%$ to $51 \%$ with acyclovir treatment [6]; another case series of 14 patients showed survival of $72 \%$ (10/14) with early initiation of acyclovir [7]. Because of its rapidly fatal course, early recognition and clinical suspicion are vital to improve clinical outcomes.

Treatment of HSV-induced fulminant hepatic failure starts first with diagnosis. Unfortunately, less than $50 \%$ of patients present with typical mucocutaneous lesions for HSV [8]. As in our patient, clinical signs include anicteric hepatitis in almost $90 \%$ of patients, wherein normal or mildly elevated bilirubin levels are accompanied by transaminase levels 100 - 1000 times normal levels often with AST > ALT. In addition, patients present with leukopenia and thrombocytopenia along with fever, flu-like symptoms and abdominal pain [9]. When trying to ascertain a diagnosis, serum HSV antibodies can often take too long to return and are often non-specific. Liver biopsy, while the gold standard for diagnosis, is often precluded by a significant coagulopathy. It has been shown that HSV DNA using PCR analysis is a useful diagnostic tool and furthermore often correlates with disease severity [9], calling for its use over non-specific HSV anti-IgM and anti-IgG.

The most commonly reported patients with HSV fulminant hepatic failure have been pregnant patients (especially in 3rd trimester) or patients who are immunosuppressed. In a case series of 137 patients by Norvell, et al., $30 \%$ were on immunosuppressive medications for liver transplantion, $23 \%$ were on immunosuppressive medications for other reasons, and 23\% were pregnant; however, in 24\%, no immunodeficiency was found. Similar to our patient, HSV hepatic failure has been reported after surgery, in one case post-thymectomy [10]. It is unclear if the pathology of HSV hepatitis is by reactivation of dormant virus or reinfection with another strain of virus. In our case, the surgery itself may have predisposed the patient to relative post-surgical immunosuppression, or the pre-operative hydrocortisone may have made him susceptible. In addition, the patient had been treated with bromocriptine, which may reduce lymphocyte reactivity and predispose to infection [11]. Because of low clinical suspicion in this presumed immunocompetent male, treatment was not initiated. 


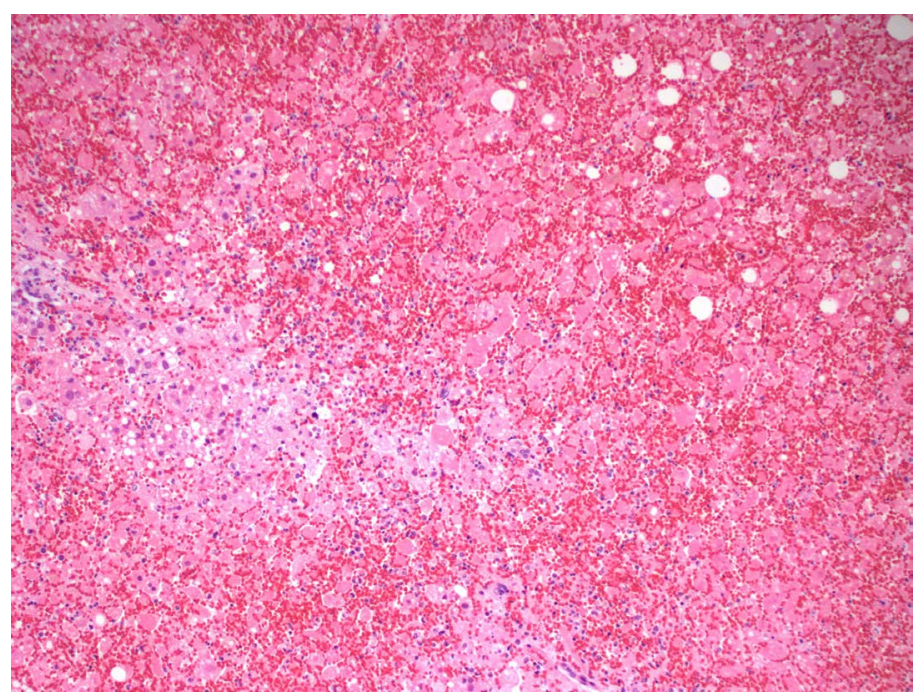

Figure 1. Low power view of liver parenchyma composed predominantly of necrotic and diffusely hemorrrhagic tissue; a focal are of viable tissue is in the left aspect of the image (hematoxylin and eosin, 40× magnification).

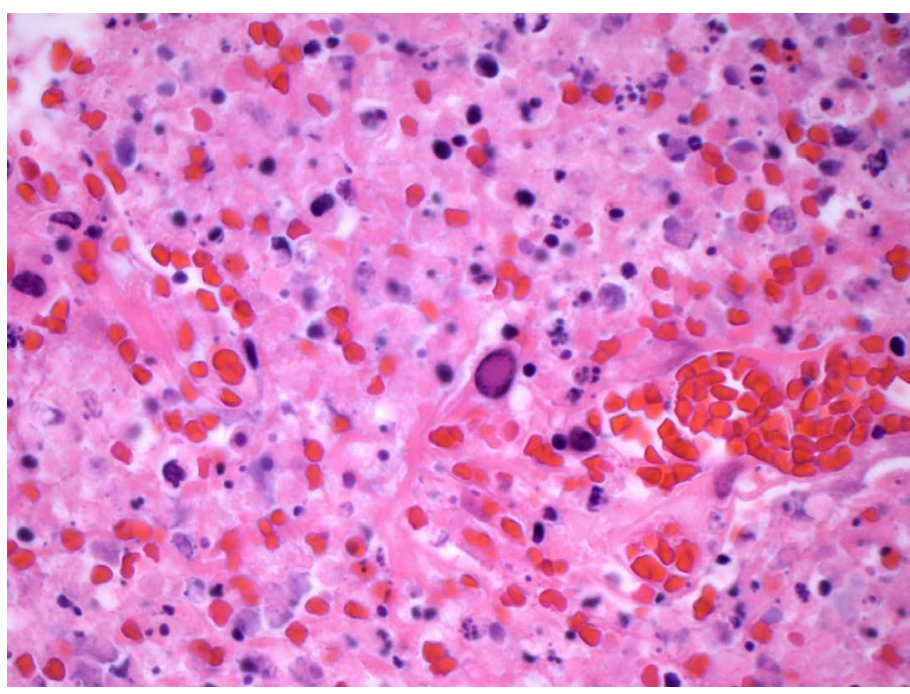

Figure 2. Medium power view of liver parenchyma demonstrating the inclusion-like ground glass change in an enlarged hepatic nucleus. The surrounding parenchyma is completely necrotic (hematoxylin and eosin, $200 \times$ magnification).

As mentioned above, treatment with acyclovir improves survival outcomes. While doses vary in case presentations, acyclovir $10 \mathrm{mg} / \mathrm{kg}$, with adjustment for renal function if necessary, has often been used. Review of the case reports shows that improved survival seems to correlate with earlier diagnosis and treatment. Given the rapidly fatal course of HSV-induced fulminant hepatic failure and the relatively low side effect profile of acyclovir, some have recommended empiric use of acyclovir in non-acetaminophen related acute liver failure patients while awaiting test results [7]. In some patients, fulminant hepatic failure progresses, and orthotopic liver transplantation is required for survival. One-year survival rates after liver transplantation have been reported to be around $40 \%$ [4]. However, because HSV sepsis is a systemic illness, patient's requiring liver transplantation from fulminant liver failure have increased risk of HSV recurrence and lifelong treatment with acyclovir is recommended [4]. However, viral resistance to acyclovir has been reported [12] leading some to suggest using foscarnet in the setting of acute liver failure in the immunosuppressed patient after OLT [4]. 


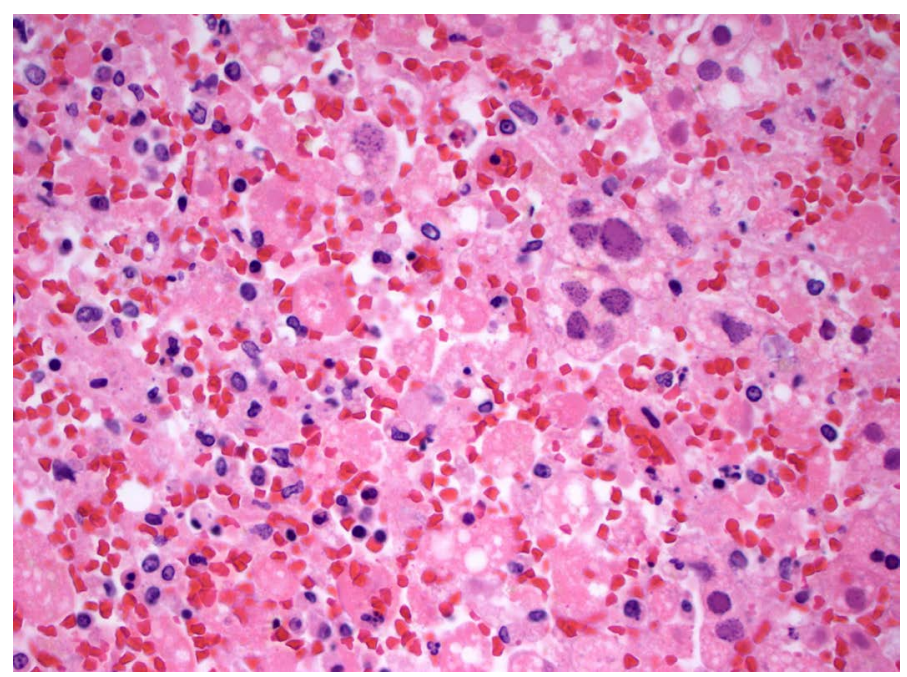

Figure 3. Medium power view of liver parenchyma demonstrating the punctate, granular chromatin in infected hepatic nuclei. The surrounding parenchyma is also completely necrotic (hematoxylin and eosin, 200× magnification).

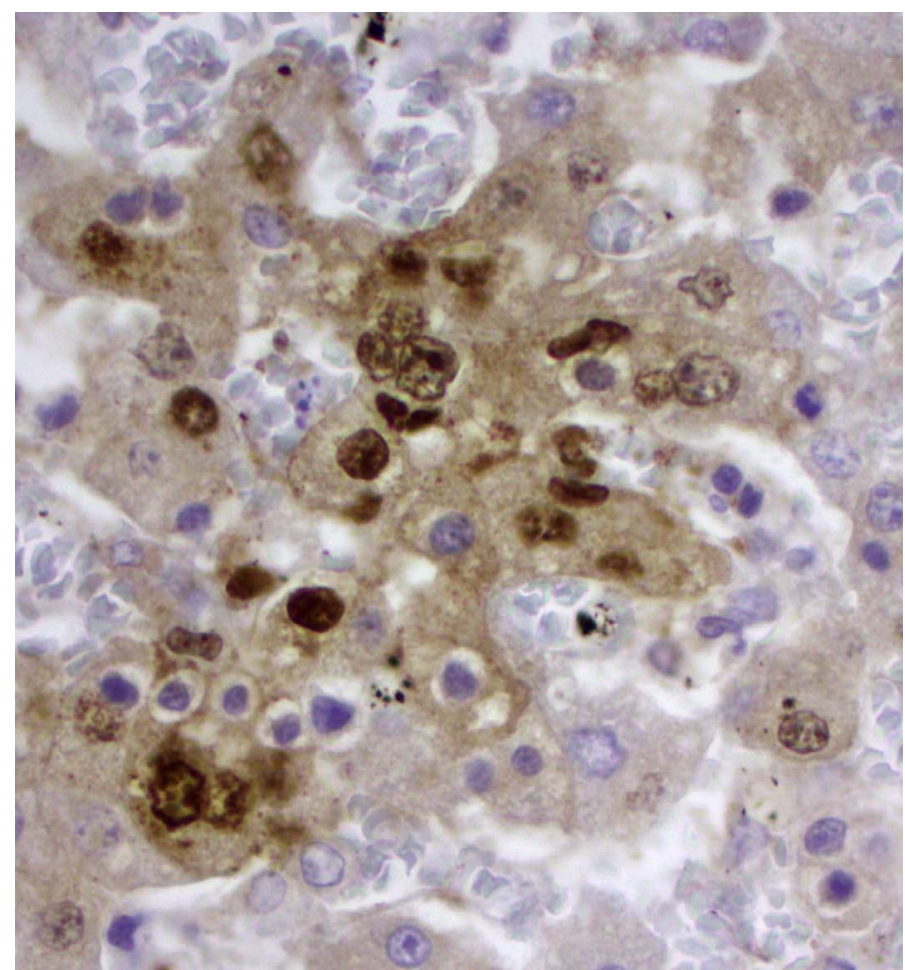

Figure 4. Medium power view of liver parenchyma stained with antiHSV antibodies demonstrating abundant virus in hepatic nuclei (dense brown) and less dense staining in hepatic cytoplasm (pale brown). The counter stain is blue, showing many nuclei in this field negative for HSV viral particles.

\section{Conclusions}

Herpes simplex fulminant hepatic failure, while rare, is rapidly fatal and is one of the treatable causes of fulminant hepatic failure. Early therapy has been shown to decrease morbidity and mortality. High clinical suspicion 
in the setting of acute liver failure is necessary, and should be especially considered in patients presenting with anicteric hepatitis. While pregnancy and immunosuppression are common risk factor characteristics, almost 1 in every 4 patients reported with HSV hepatitis is immunocompetent. Because of its improvement in clinical outcomes, and low side effect profile and cost, IV acyclovir should be considered empirically in all patients presenting with non-acetaminophen acute liver failure.

Pathology Teaching Point: Although the viral cytopathic effects of herpes simplex virus infection are quite characteristic, in some cases, extensive necrosis or balloon degeneration of hepatocytes can make the diagnosis more challenging. In such cases, confirmation with an immunohistochemical stain directed at HSV can be very helpful. Other viruses within the herpesvirus family can also elicit distinctive cytopathic changes, such as the CMV inclusion body, and again, immunohistochemical stains can be very helpful in distinguishing specific viral etiologies. In the absence of immunohistochemistry, other diagnostic tests may be necessary (such as PCR) to identify the causative agent.

Critical Care Teaching Point: In critically ill patients, diagnostic and therapeutic measures often must occur simultaneously, not sequentially. This case shows the importance of considering even rare causes of acute deterioration, in this case HSV hepatitis. While aggressive supportive care is critical, initiation of broad-based therapy allows multiple possible etiologies to be treated while diagnostic studies pending. In the patient who has a rare disease, the incidence of that disease is $100 \%$.

\section{References}

[1] Xu, F., Sternberg, M.R., Kottiri, B.J., et al. (2006) Trends in Herpes Simplex Virus Type 1 and Type 2 Seroprevalence in the United States. JAMA, 296, 964-973. http://dx.doi.org/10.1001/jama.296.8.964

[2] Schiodt, F.D.T., Shakil, O., McGuire, B., Samuel, G. and Lee, W., The Acute Liver Failure Study Group (2003) Viral Hepatitis-Related Acute Liver Failure. American Journal of Gastroenterology, 98, 448-453. http://dx.doi.org/10.1016/S0002-9270(02)05891-4

[3] Flewett, T.H., Parker, R.G. and Philip, W.M. (1969) Acute Hepatitis Due to Herpes Simplex Virus in an Adult. Journal of Clinical Pathology, 22, 60-66. http://dx.doi.org/10.1136/jcp.22.1.60

[4] Riediger, C., Sauer, P., Matevossian, E., Muller, M.W., Buchler, P. and Friess, H. (2009) Herpes Simplex Virus Sepsis and Acute Liver Failure. Clinical Transplantation, 23, 37-41. http://dx.doi.org/10.1111/j.1399-0012.2009.01108.x

[5] Aboguddah, A., Stein, H.B., Phillips, P., Amar, J. and English, R. (1991) Herpes Simplex Hepatitis in a Patient with Psoriatic Arthritis Taking Prednisone and Methotrexate. Report and Review of the Literature. Journal of Rheumatology, 18, 1406-1412.

[6] Norvell, J., Blei, A., Jovanovic, B. and Levitsky, J. (2007) Herpes Simplex Virus Hepatitis: An Analysis of the Published Literature and Institutional Cases. Liver Transplantation, 13, 1428-1434. http://dx.doi.org/10.1002/lt.21250

[7] Peters, D., Greene, W., Ruggiero, F. and McGarrity, T. (2000) Herpes Simplex-Induced Fulminant Hepatitis in Adults: A Call for Empiric Therapy. Digestive Diseases and Sciences, 45, 2399-2404. http://dx.doi.org/10.1023/A:1005699210816

[8] Farr, R.W., Short, S. and Weissman, D. (1997) Fulminant Hepatitis during Herpes Simplex Virus Infection in Apparently Immunocompetent Adults: Report of Two Cases and Review of the Literature. Clinical Infectious Diseases, 24, 1191-1194. http://dx.doi.org/10.1086/513646

[9] Beersma, M.F.C., Verjans, G.M., van Doornum, G.J., et al. (2011) Quantification of Viral DNA and Liver Enzymes in Plasma Improves Early Diagnosis and Management of Herpes Simplex Virus Infection. Journal of Viral Hepatitis, 18, 160-166. http://dx.doi.org/10.1111/j.1365-2893.2010.01352.x

[10] Biancofiore, G., Bisa, M., Bindi, L.M., et al. (2007) Liver Transplantation Due to Herpes Simplex Virus-Related Sepsis Causing Massive Hepatic Necrosis after Thoracoscopic Thymectomy. Minerva Anestesiologica, 73, 319-322.

[11] Hiestand, P.C., Mekler, P., Nordmann, R., et al. (1986) Prolactin as a Modulator of Lymphocyte Responsiveness Provides a Possible Mechanism of Action for Cyclosporine. Proceedings of the National Academy of Sciences of the United States of America, 83, 2599. http://dx.doi.org/10.1073/pnas.83.8.2599

[12] Longerich, T., Eisenbach, C., Penzel, R., et al. (2005) Recurrent Herpes Simplex Virus Hepatitis after Liver Retransplantation Despite Acyclovir Therapy. Liver Transplantation, 11, 1289-1294. http://dx.doi.org/10.1002/lt.20567 
Scientific Research Publishing (SCIRP) is one of the largest Open Access journal publishers. It is currently publishing more than 200 open access, online, peer-reviewed journals covering a wide range of academic disciplines. SCIRP serves the worldwide academic communities and contributes to the progress and application of science with its publication.

Other selected journals from SCIRP are listed as below. Submit your manuscript to us via either submit@scirp.org or Online Submission Portal.
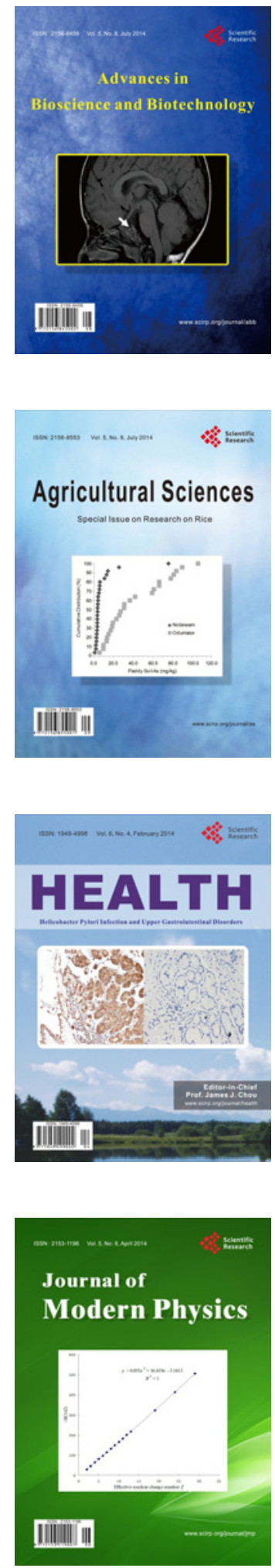
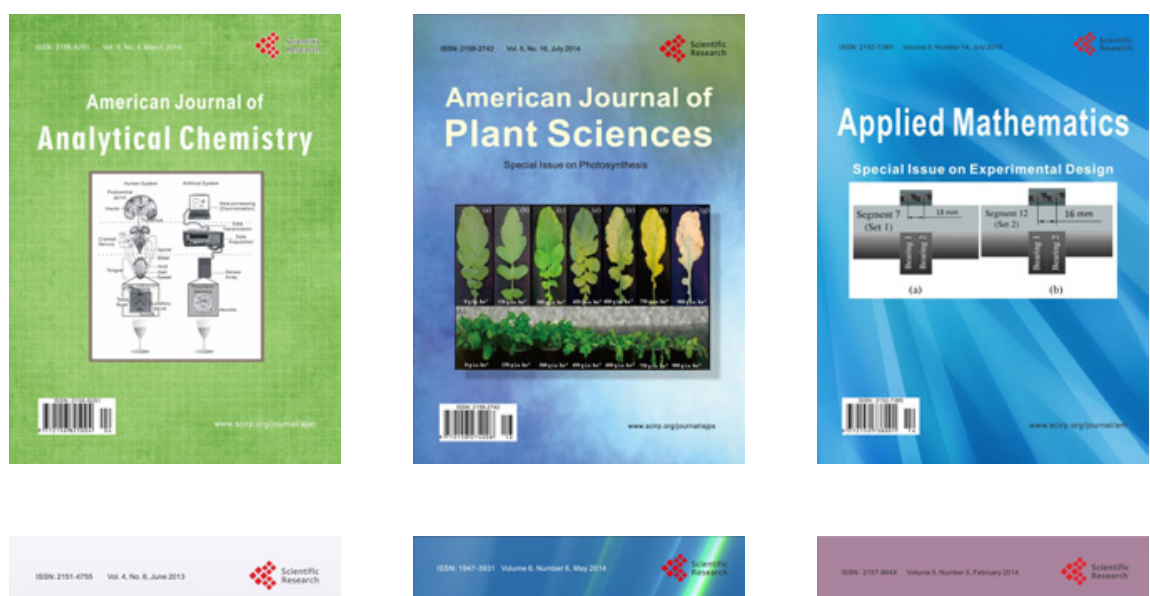

Creative Education
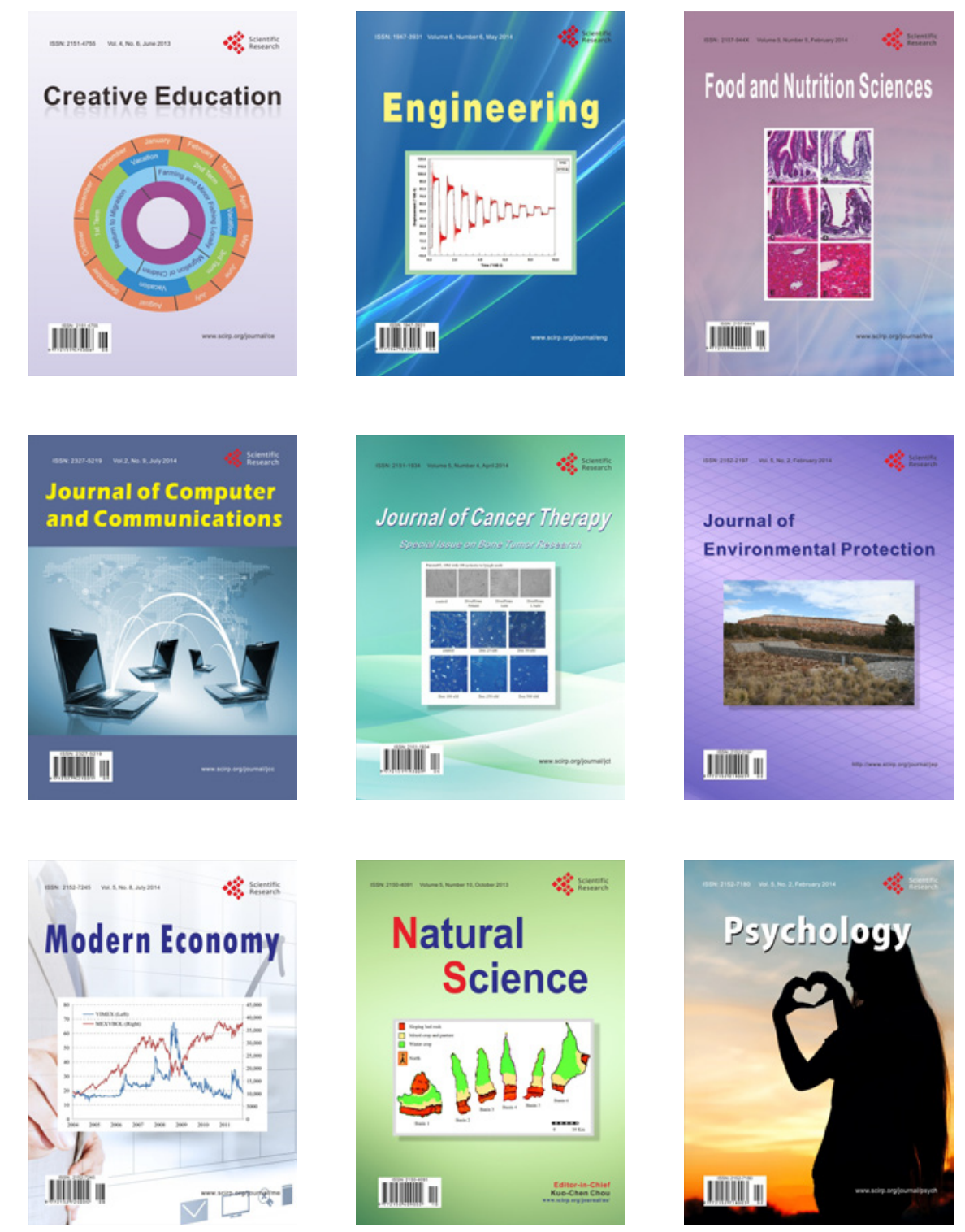